\title{
A universal curve of optimum thermoelectric figures of merit for bulk and low-dimensional semiconductors
}

\author{
Nguyen T. Hung ${ }^{*}$ Ahmad R. T. Nugraha, and Riichiro Saito \\ Department of Physics, Tohoku University, Sendai 980-8578, Japan
}

\begin{abstract}
Analytical formulas for thermoelectric figure of merit and power factor are derived based on the one-band model. We find that there is a direct relationship between the optimum figures of merit and the optimum power factors of semiconductors despite of the fact that the two quantities are generally given by different values of chemical potentials. By introducing a dimensionless parameter consisting of optimum power factor and lattice thermal conductivity (without electronic thermal conductivity), it is possible to unify optimum figures of merit of both bulk and low-dimensional semiconductors into a single universal curve that covers lots of materials with different dimensionalities.
\end{abstract}

\section{INTRODUCTION}

Most of electrical energy that we consume in daily life comes from thermal processes, such as heat engines in cars and power plants, in which more than half of the energy is wasted in form of heat [1]. Research on thermoelectricity for recovering this waste heat, i.e. to convert the waste heat directly into electric energy, is thus of great interest [1, 2]. A good thermoelectric (TE) material is characterized by how efficient the electricity can be obtained for a given heat source, in which the thermoelectric figure of merit $Z T=S^{2} \sigma \kappa^{-1} T$ is usually evaluated, where $S, \sigma, \kappa$, and $T$ are the Seebeck coefficient, the electrical conductivity, the thermal conductivity, and the average absolute temperature, respectively. It is wellknown that obtaining the optimum $Z T$ (or shortly $Z T_{\text {opt }}$ ) for a certain TE material, where $Z T_{\text {opt }}$ is defined as the maximum value of $Z T$ as a function of the chemical potential, is often complicated by the interdependence of $S, \sigma$, and $\kappa[3]$. Therefore, finding the best material to obtain as large $Z T_{\text {opt }}$ as possible has been a great challenge for many years. As one strategy, using lowdimensional semiconductors with a large density of states at the top of the valence band (or the bottom of the conduction band) was suggested by Hicks and Dresselhaus to improve $Z T_{\text {opt }}$ 4 6]. However, we recently pointed out that in terms of their power factor $\mathrm{PF}=S^{2} \sigma$, only low-dimensional semiconductors with confinement length smaller than thermal de Broglie wavelength prove to be useful TE materials compared with the bulk ones [7].

Another strategy to find the best thermoelectric materials is by defining a material parameter that can be the most essential one to determine $Z T_{\text {opt }}$. We can mention several efforts by researchers in the past who proposed some parameters for evaluating $Z T_{\text {opt }}$. For example, in 1996, Mahan and Sofo introduced a dimensionless material parameter $k_{B} T / E_{b}$ [8], where $k_{B}$ and $E_{b}$, are the Boltzmann constant and the energy band width, respectively. When $E_{b}$ is infinitesimal, the transport distribution function $\mathcal{T}=v^{2} \tau \mathcal{D}$ forms a delta function that leads

\footnotetext{
* nguyen@flex.phys.tohoku.ac.jp
}

to the largest possible value of $Z T_{\text {opt }}$, where $v$ is the carrier velocity, $\tau$ is the carrier relaxation time, and $\mathcal{D}$ is the density of states of the carrier at the Fermi energy. This work was revisited from a Landauer perspective by Jeong et al. 9], they found that a finite $E_{b}$ dispersion produces a higher $Z T$ when the lattice thermal conductivity is finite. Much earlier, in 1959, Chasmar and Stratton suggested that a parameter $B=5.745 \times 10^{-6}\left(\mu / \kappa_{l}\right)\left(m / m_{0}\right)^{3 / 2} T^{5 / 2}$, where $\mu, \kappa_{l}, m$, and $m_{0}$ are the carrier mobility, the lattice thermal conductivity, the carrier effective mass, and the free electron mass, respectively, determines the optimum $Z T$ [10]. Note that the product of $\mu$ and $\left(m / m_{0}\right)^{3 / 2}$ was commonly called weighted mobility. A large $B$ usually corresponds to a high $Z T$ value at a certain chemical potential. The advantage of the parameter $B$ is that to obtain a good TE material, instead of checking all the interdependent transport properties, one should look for a semiconductor with a high weighted mobility and a low lattice thermal conductivity $\kappa_{l}$, which are less dependent on each other. Although $E_{b}$ and $B$ have been used to guide researches in thermoelectricity for many years, it is not possible to directly identify $Z T_{\text {opt }}$ by using only these parameters. On the other hand, there have been a lot of efforts dedicated to optimize the PF, giving the optimum power factor $\mathrm{PF}_{\text {opt }}$ that can be obtained by changing the chemical potential [11. Since $Z T_{\text {opt }}$ generally occurs at a different chemical potential from $\mathrm{PF}_{\text {opt }}$, i.e., $Z T_{\text {opt }} \neq \mathrm{PF}_{\text {opt }} \kappa^{-1} T$, one always needs to measure or estimate $Z T_{\text {opt }}$ independently from $\mathrm{PF}_{\text {opt }}$ by checking again chemical potential dependence of $Z T$. Therefore, it should be useful for thermoelectric applications if we can calculate $Z T_{\text {opt }}$ from the information of $\mathrm{PF}_{\text {opt }}$ or other simple parameters.

In this paper, we propose that a new material parameter $\alpha=\left(\mathrm{PF}_{\text {opt }} / \kappa_{l}\right) T$ can be defined to directly determine $Z T_{\text {opt }}$. Although, $Z T_{\text {opt }}$ and $\mathrm{PF}_{\text {opt }}$ are generally optimized at different chemical potentials, the value of $Z T_{\text {opt }}$ can be calculated using an analytical formula that involves the so-called Lambert $W$ function, where $\alpha$ can be used as an input parameter. Without losing generality, the analytical formula for $Z T_{\text {opt }}$ is derived within the one-band model and nondegenerate semiconductor approximation. We will show that $Z T_{\text {opt }}$ for both bulk and low-dimensional semiconductors can be unified into 
a single universal curve, which allows us to predict and understand the materials of different dimensions that can have better $Z T_{\text {opt }}$ by simply calculating the $\alpha$ parameter.

The rest of this paper is organized as follows. In Sec. II] we start the derivation of some formulas of thermoelectric properties from the conventional Boltzmann transport theory. This initial derivation will give us PF and $Z T$ formulas involving integrals that must be calculated numerically. In Sec. III. we apply a non-degenerate semiconductor approximation so that $\mathrm{PF}_{\text {opt }}$ and $Z T_{\text {opt }}$ can be obtained analytically, which results in the universal curve of $Z T_{\text {opt. }}$ Finally, in Sec. IV] we conclude the paper and give a few perspectives for future works in the field of thermoelectricity. We also provide some appendixes for additional information about the derivation of the formulas and the Lambert $W$ function.

\section{THEORETICAL METHODS}

By solving the linearized Boltzmann equations within the one-band model and the relaxation time approximation, three TE transport properties are related to the transport distribution function $\mathcal{T}(E)$ as follows:

$$
\sigma=q^{2} \mathcal{L}_{0}, \quad S=\frac{1}{q T} \frac{\mathcal{L}_{1}}{\mathcal{L}_{0}}, \quad \kappa_{e}=\frac{1}{T}\left(\mathcal{L}_{2}-\frac{\mathcal{L}_{1}^{2}}{\mathcal{L}_{0}}\right)
$$

where $\sigma, S, \kappa_{e}$, are the electrical conductivity, the Seebeck coefficient, the electronic thermal conductivity, respectively. $\mathcal{L}_{i}$ is the transport integral that is defined by 8

$$
\mathcal{L}_{i}=\int \mathcal{T}(E)\left(E-E_{F}\right)^{i}\left(-\frac{\partial f_{0}}{\partial E}\right) \mathrm{d} E, i=0,1,2,
$$

where $E$ is the energy of carrier, $f_{0}=1 /\left[e^{\left(E-E_{F}\right) / k_{B} T}+1\right]$ is the Fermi-Dirac distribution function, where the Fermi energy $E_{F}$ is defined as the chemical potential measured from the bottom (top) of the conduction (valence) energy band in an n-type (p-type) semiconductor, and $\mathcal{T}(E)$ is defined

$$
\mathcal{T}(E)=v_{x}^{2}(E) \tau(E) \mathcal{D}(E),
$$

where $v_{x}(E), \tau(E)$, and $\mathcal{D}(E)$ are the group velocity in the $x$ direction, the relaxation time, and the density of states (DOS) of the carrier, respectively.

From Eqs. (1) and (2), the thermoelectric power factor $\mathrm{PF}$ and figure of merit $Z T$ can be written as

$$
\begin{aligned}
& \mathrm{PF}=S^{2} \sigma=\frac{1}{T^{2}} \frac{\mathcal{L}_{1}^{2}}{\mathcal{L}_{0}} \\
& Z T=\frac{S^{2} \sigma}{\kappa_{e}+\kappa_{l}} T=\beta \frac{\mathcal{L}_{1}^{2}}{\mathcal{L}_{0} \mathcal{L}_{2}-\mathcal{L}_{1}^{2}},
\end{aligned}
$$

where $\kappa_{l}$ is the lattice thermal conductivity and $\beta=$ $1 /\left(\kappa_{l} / \kappa_{e}+1\right) \leq 1$. It is clear from Eqs. (4) and (5) that the $\mathrm{PF}$ and $Z T$ have different dependence on $E_{F}$.
For the sake of simplicity, we consider a single parabolic band, in which the energy band structure and the group velocity can be given as $E(\mathbf{k})=\hbar^{2} \mathbf{k}^{2} / 2 m$ and $v(\mathbf{k})=\frac{1}{\hbar}[\partial E(\mathbf{k}) / \partial \mathbf{k}]=\hbar \mathbf{k} / m$, respectively, where $\mathbf{k}$ is the wave vector of the carrier, $m$ is the carrier effective mass, and $\hbar$ is the Planck constant. We assumed that the material is isotropic with a certain dimension $d=1,2,3$, the group velocity $v_{x}^{2}(E)=v^{2}(\mathbf{k}) / d=\hbar^{2} \mathbf{k}^{2} / m^{2} d=$ $2 E / m d$, and the carrier relaxation time is inversely proportional to the carrier DOS [12], $\tau(E)=C \mathcal{D}^{-1}(E)$, where $C$ is the scattering coefficient in units of $\mathrm{W}^{-1} \mathrm{~m}^{-3}$. The DOS is defined as $\mathcal{D}(E)=\frac{2}{\Omega} \sum_{\mathbf{k}} \delta[E-E(\mathbf{k})]$ in units of $\mathrm{J}^{-1} \mathrm{~m}^{-3}$, where the factor 2 accounts for the spin degeneracy and $\Omega$ is the volume of the system. Detailed derivations of how we can calculate $C$ for a typical material are given in Appendix A. After substituting $v_{x}^{2}(E)$ and $\tau(E)$ into $\mathcal{T}(E)$ in Eq. (3), the integrals $\mathcal{L}_{i}$ in Eq. (2) can be written as

$$
\begin{aligned}
\mathcal{L}_{0} & =\frac{2 C}{m d}\left(k_{B} T\right) F_{0}, \\
\mathcal{L}_{1} & =\frac{2 C}{m d}\left(k_{B} T\right)^{2}\left(2 F_{1}-\eta F_{0}\right), \\
\mathcal{L}_{2} & =\frac{2 C}{m d}\left(k_{B} T\right)^{3}\left(3 F_{2}-4 \eta F_{1}+\eta^{2} F_{0}\right),
\end{aligned}
$$

where $\eta=E_{F} / k_{B} T$ is the reduced (or dimensionless) chemical potential and $F_{j}(\eta)=\int \eta^{j} f_{0} \mathrm{~d} \eta$ is the FermiDirac integral. By substituting $\mathcal{L}_{i}$ in Eqs. (6), (7), and (8) into Eqs. (4) and (5), we obtain the formulas of the PF and $Z T$ as follows:

$$
\begin{aligned}
\mathrm{PF} & =\frac{2 C k_{B}^{3} T}{m d} \frac{\left(2 F_{1}-\eta F_{0}\right)^{2}}{F_{0}} \\
Z T & =\beta \frac{\left(2 F_{1}-\eta F_{0}\right)^{2}}{F_{0}\left(3 F_{2}-4 \eta F_{1}+\eta^{2} F_{0}\right)-\left(2 F_{1}-\eta F_{0}\right)^{2}},
\end{aligned}
$$

where the integrals $F_{0}, F_{1}$, and $F_{2}$ are calculated numerically.

\section{RESULTS AND DISCUSSION}

In this section we firstly discuss an example of calculating the $\mathrm{PF}$ and $Z T$ as a function of $\eta$ for one semiconducting material by using Eqs. (9) and (10) numerically. After that, we simplify the PF and $Z T$ formulas by considering nondegenerate semiconductor approximation, which gives us analytical formulas of $\mathrm{PF}_{\mathrm{opt}}$ and $Z T_{\mathrm{opt}}$. The $Z T_{\text {opt }}$ formula can then be plotted and compared with various experimental data, leading to a universal curve of $Z T_{\mathrm{opt}}$.

\section{A. Example of a typical material}

Figures 1(a)-(d) show, respectively, the dependence of $S$ and $\sigma$, the PF [Eq. (9)], $\beta$ and $\kappa_{l} / \kappa_{e}$, and $Z T$ [Eq. (10] ] 

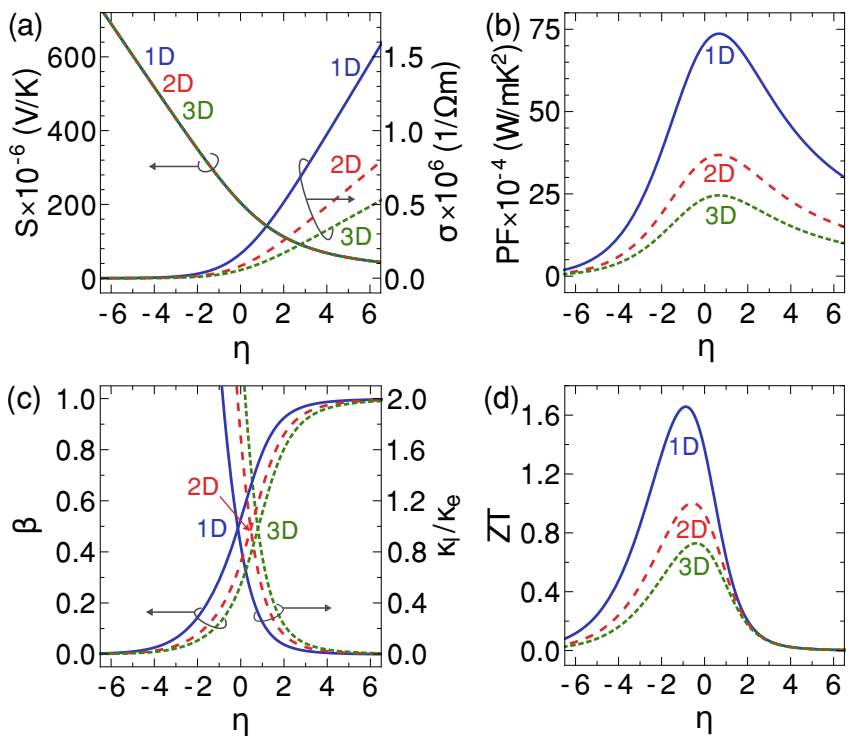

FIG. 1. (a) $S$ and $\sigma$, (b) PF, (c) $\beta$ and $\kappa_{l} / \kappa_{e}$, and (d) $Z T$ as a function of the reduced chemical potential $\eta$ for the $1 \mathrm{D}$, $2 \mathrm{D}$, and 3D systems, respectively. The carrier effective mass, the carrier mobility, and the lattice thermal conductivity are set to be $m=1.12 m_{0}, \mu=173 \mathrm{~cm}^{2} / \mathrm{Vs}$, and $\kappa_{l}=0.728$ $\mathrm{W} / \mathrm{mK}$, respectively, for n-type $\mathrm{Bi}_{2} \mathrm{Te}_{2.7} \mathrm{Se}_{0.3}$ at room temperature $(T=298 \mathrm{~K})$ [13.

on the reduced chemical potential $\eta$ for different dimensions. When plotting Figs 1(a)-(d), we consider a typical semiconductor, n-type $\mathrm{Bi}_{2} \mathrm{Te}_{2.7} \mathrm{Se}_{0.3}$, at $T=298 \mathrm{~K}$ and the doping concentration about $0.92 \times 10^{19} \mathrm{~cm}^{-3}$. The carrier effective mass, carrier mobility, lattice thermal conductivity are taken to be $m=1.12 m_{0}, \mu=173$ $\mathrm{cm}^{2} / \mathrm{Vs}$, and $\kappa_{l}=0.728 \mathrm{~W} / \mathrm{mK}$, respectively, for the 3D $(d=3)$ bulk n-type $\mathrm{Bi}_{2} \mathrm{Te}_{2.7} \mathrm{Se}_{0.3}[13$. The scattering coefficient $C=1.18 \times 10^{33} \mathrm{~W}^{-1} \mathrm{~m}^{-3}$ is obtained from $m$ and $\mu$ by using Eq. A13 in Appendix A, which leads to an average relaxation time of about $0.1 \mathrm{ps}$. We temporarily use the same parameter values of $m, \kappa_{l}$, and $C$ for the 1D $(d=1)$ and $2 \mathrm{D}(d=2)$ systems as the 3D's. However, these parameters generally vary by dimensions for different materials, as we adopt later in Sec. IIIC

Figure 1(a) shows that $S$ is independent of $d$ and it increases with decreasing $\eta$, while $\sigma$ depends on $d$ and it decreases with decreasing $\eta$. This behavior can be understood in terms of their units since the units $[\mathrm{V} / \mathrm{K}]$ of $S$ show no dependence of length scale, while the unit $[1 / \Omega \mathrm{m}]$ of $\sigma$ show dependence of length scale. Figure 1(b) shows a strong enhancement of the maximum PF around $\eta \approx 0$ in the low-dimensional systems (1D and 2D). For the bulk (3D) system, the theoretical maximum $\mathrm{PF}$ value is about $0.0025 \mathrm{~W} / \mathrm{mK}^{2}$, which is in a good agreement with the experimental data of about $0.0021 \mathrm{~W} / \mathrm{mK}^{2}$ [13]. In the case of $\eta \gg 0$, we can see that $S$ approaches zero because the system becomes metallic at high doping concentrations, while $\sigma$ is close to zero when $\eta \ll 0$ [Fig. 1(a)]. Therefore, the $\mathrm{PF}_{\text {opt }}$ occurs at $\eta \approx 0$, in
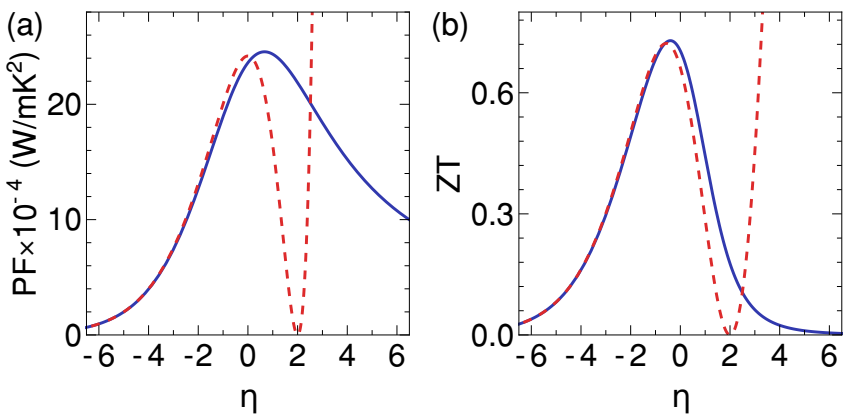

FIG. 2. (a) PF and (b) $Z T$ as functions of the reduced chemical potential $\eta$. Results from the formulas involving numerical integrations and those from analytical calculation (nondegenerate semiconductor approximation) are represented by solid and dashed lines, respectively. The carrier effective mass, the carrier mobility, and the lattice thermal conductivity are set to be $m=1.12 m_{0}, \mu=173 \mathrm{~cm}^{2} / \mathrm{Vs}$, and $\kappa_{l}=0.728 \mathrm{~W} / \mathrm{mK}$, respectively, for $3 \mathrm{D}$ n-type $\mathrm{Bi}_{2} \mathrm{Te}_{2.7} \mathrm{Se}_{0.3}$ at room temperature [13].

which $E_{F}$ lies at the bottom (top) of conduction (valence) energy band in a p-type (n-type) semiconductor, for all the 1D, 2D, and 3D systems, as shown in Fig. 1(b). Figure 1(d) shows a strong enhancement of the maximum $Z T$ values in the $1 \mathrm{D}$ and $2 \mathrm{D}$ systems, which is known as the Hicks-Dresselhaus theory [4, 5]. For the 3D system, the theoretical maximum $Z T$ value is about 0.72 , which is in a good agreement with the experimental data of about 0.73 13. In the case of $\eta \gg 0$, the coefficient $\beta=1 /\left(\kappa_{l} / \kappa_{e}+1\right) \approx 1$ since $k_{e}$ is much larger than $k_{l}$ when the system is metallic, as shown in Fig. 1(c). In contrast, $\beta \approx 0$ when $\eta \ll 0$ because $k_{e}$ is near zero (few free electron carriers in the insulators) [see Fig. 1(c)]. Therefore, $Z T_{\text {opt }}$ is found at $\eta<0$, in which $E_{F}$ lies in the energy gap, as shown in Fig. 1(d). Important information in Figs. 1 (b) and (d) is that the PF and $Z T$ are optimized at $\eta \approx 0$ and $\eta<0$, respectively, for all $1 \mathrm{D}, 2 \mathrm{D}$, and $3 \mathrm{D}$ systems, although the two quantities are located at different $\eta$ for each $d$.

\section{B. Nondegenerate semiconductor approximation}

Next, we would like to obtain the analytical formulas for both the $\mathrm{PF}_{\mathrm{opt}}$ and $Z T_{\mathrm{opt}}$. In Eqs. (9) and (10), which were used to plot Figs. 1(b) and 1(d), we have considered the full solutions of Fermi-Dirac integrals $F_{0}, F_{1}$, and $F_{2}$ numerically. The problem is how can we get analytical formulas for $\mathrm{PF}_{\mathrm{opt}}$ and $Z T_{\mathrm{opt}}$ to approach these two quantities? Since $\mathrm{PF}_{\mathrm{opt}}\left(Z T_{\mathrm{opt}}\right)$ is optimized at $\eta \approx 0$ $(\eta<0)$, we may use the nondegenerate semiconductor approximation that is especially valid for $\eta \leq 0$ [14. In this case, the Fermi-Dirac integral is approximated as $F_{j}(\eta) \approx e^{\eta} \Gamma(j+1)$ [14], where $\Gamma(j)$ is the Gamma function. By substituting $F_{0}=e^{\eta}, F_{1}=e^{\eta}$, and $F_{2}=2 e^{\eta}$ 
into Eq. (9), we get the PF formula as

$$
\mathrm{PF}=\frac{2 C k_{B}^{3} T}{m d}(2-\eta)^{2} e^{\eta} .
$$

Since $\kappa_{e}=\frac{1}{T}\left(\mathcal{L}_{2}-\mathcal{L}_{1}^{2} / \mathcal{L}_{0}\right)=4 C k_{B}^{3} T^{2} e^{\eta} /(m d)$ [see Eq. (1)], $\beta$ can be written as

$$
\beta=\frac{1}{\left[2 /\left(\alpha e^{\eta}\right)\right]+1},
$$

where

$$
\alpha=\frac{8 C k_{B}^{3} T^{2}}{m d \kappa_{l}}
$$

is a dimensionless parameter. Substituting $\beta$ into Eq. 10 and applying the approximation of $F_{j}$, we obtain

$$
Z T=\frac{(2-\eta)^{2}}{\left[4 /\left(\alpha e^{\eta}\right)\right]+2} .
$$

In Figs. 2(a) and 2(b), we respectively show $\mathrm{PF}_{\text {opt }}$ and $Z T_{\text {opt }}$ that are calculated based on the full solutions of Fermi-Dirac integrals [Eqs. (9) and (10)] and the nondegenerate semiconductor approximation [Eqs. (11) and (14)]. If we just focus on the values of $\mathrm{PF}_{\text {opt }}$ and $Z T_{\text {opt }}$ (local maxima of PF and $Z T$ ) at $\eta \leq 0$, we can see that the analytical formulas based on the nondegenerate semiconductor approximation can nicely reproduce the $\mathrm{PF}_{\text {opt }}$ and $Z T_{\text {opt }}$ of the full solutions. Therefore, we can determine the $\mathrm{PF}_{\text {opt }}$ and $Z T_{\text {opt }}$ from Eqs. (11) and 14 by solving $\mathrm{d}(\mathrm{PF}) / \mathrm{d} \eta=0$ and $\mathrm{d}(Z T) / \mathrm{d} \eta=0$, respectively. The formulas obtained for $\mathrm{PF}_{\text {opt }}$ and $Z T_{\text {opt }}$ are

$$
\mathrm{PF}_{\mathrm{opt}}=\frac{8 C k_{B}^{3} T}{m d}, \quad Z T_{\mathrm{opt}}=\frac{W_{0}^{2}(\alpha)}{2}+W_{0}(\alpha),
$$

where $W_{0}(\alpha)$ is the principal branch of the Lambert $W$ function (see Appendix B). By substituting the $\mathrm{PF}_{\text {opt }}$ in Eq. (15) into Eq. (13), the $\alpha$ parameter is now expressed in terms of the $\mathrm{PF}_{\text {opt }}$ and $\kappa_{l}$,

$$
\alpha=\frac{\mathrm{PF}_{\mathrm{opt}}}{\kappa_{l}} T \text {. }
$$

The corresponding reduced chemical potentials for the $\mathrm{PF}_{\mathrm{opt}}$ and $Z T_{\mathrm{opt}}$ are $\eta_{\mathrm{opt}}^{\mathrm{PF}}=0$ and $\eta_{\mathrm{opt}}^{Z T}=-W_{0}(\alpha)$, respectively [see Fig. 2]. Based on the simple analytical formulas in Eq. 15, the values of the $\mathrm{PF}_{\text {opt }}$ and $Z T_{\text {opt }}$ can be calculated directly from $C, d, m$, $\kappa_{l}$, and $T$, which could be measured in experiments. For example, in the case of $3 \mathrm{D}$ n-type $\mathrm{Bi}_{2} \mathrm{Te}_{2.7} \mathrm{Se}_{0.3}$ at room temperature, taken from Ref. 13, we have $C=1.18 \times 10^{33} \mathrm{~W}^{-1} \mathrm{~m}^{-3}$ (see also Appendix A), $d=3$, $m=1.12 m_{0}, \kappa_{l}=0.728 \mathrm{~W} / \mathrm{mK}$, and hence $\mathrm{PF}_{\text {opt }}=$ $0.0024 \mathrm{~W} / \mathrm{mK}^{2}$ and $Z T_{\text {opt }}=0.72$. This analytical result agrees well with both fully numerical calculation $\left(\mathrm{PF}_{\text {opt }}=0.0025 \mathrm{~W} / \mathrm{mK}^{2}\right.$ and $\left.Z T_{\text {opt }}=0.72\right)$ [see Fig. 2 and experimental data $\left(\mathrm{PF}_{\mathrm{opt}}=0.0021 \mathrm{~W} / \mathrm{mK}^{2}\right.$ and $\left.Z T_{\text {opt }}=0.73\right)[13$.

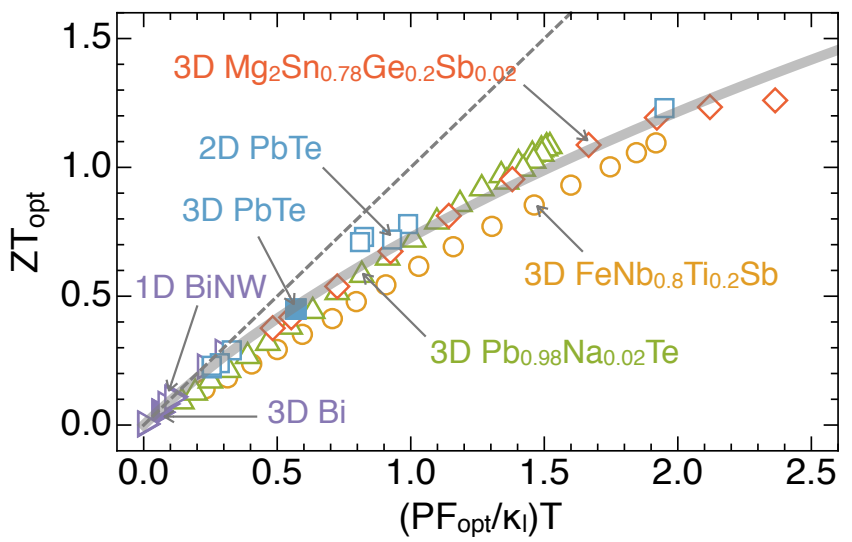

FIG. 3. $Z T_{\text {opt }}$ as a function of $\alpha=\left(\mathrm{PF}_{\text {opt }} / \kappa_{l}\right) T$. The solid line denotes the theoretical curve from Eq. (15), while the dashed line is the plot of $Z T_{\mathrm{opt}}=\alpha$ as a guide for eyes. The symbols represent experimental results of $1 \mathrm{D} \mathrm{Bi}$ nanowires $(\triangleright)$ and 3D $\mathrm{Bi}(\triangleright)$ [16], 2D PbTe quantum wells $(\square)$ and 3D $\mathrm{PbTe}(\boldsymbol{\square})$ 17, $3 \mathrm{D} \mathrm{Pb} 0.98 \mathrm{Na}_{0.02} \mathrm{Te}(\triangle)$ 18, $3 \mathrm{D} \mathrm{FeNb}_{0.8} \mathrm{Ti}_{0.2} \mathrm{Sb}$ (॰) 19], and $3 \mathrm{D} \mathrm{Mg}_{2} \mathrm{Sn}_{0.78} \mathrm{Ge}_{0.2} \mathrm{Sb}_{0.02}(\diamond)$ [20], respectively.

To gain insight into the $\mathrm{PF}_{\text {opt }}$, we can substitute the coefficient $C$ in Eq. A13 from Appendix A to the $\mathrm{PF}_{\text {opt }}$ formula in Eq. (15), so that the $\mathrm{PF}_{\text {opt }}$ is given by

$$
\mathrm{PF}_{\mathrm{opt}}=\frac{16 \mu k_{B}^{2}}{q L^{3}}\left(\frac{L}{\Lambda}\right)^{d} \frac{\Gamma\left(\frac{5}{2}\right)}{\Gamma\left(\frac{7-d}{2}\right) \Gamma\left(\frac{d}{2}\right)},
$$

where $L$ is the confinement length for a particular material dimension, and $\Lambda=\left[2 \pi \hbar^{2} /\left(m k_{B} T\right)\right]^{1 / 2}$ is the thermal de Broglie wavelength (a measure of the thermodynamic uncertainty for the localization of a electron or hole of mass $m$ ) [15]. Equation (17) shows the dependence of the $\mathrm{PF}_{\text {opt }}$ on $\mu, d, L$, and $\Lambda$. By scaling the $\mathrm{PF}_{\text {opt }}$ with the optimum $\mathrm{PF}$ of a $3 \mathrm{D}$ system, i.e. $\mathrm{PF}_{\mathrm{opt}}^{3 \mathrm{D}}$, we find that the ratio $\mathrm{PF}_{\text {opt }} / \mathrm{PF}_{\text {opt }}^{3 \mathrm{D}}$ merely depends on the factor $(L / \Lambda)^{d-3}$, consistent with our previous work [7]. It is clear that the $\mathrm{PF}_{\text {opt }}$ is enhanced for $1 \mathrm{D}$ and $2 \mathrm{D}$ semiconductors only when $L$ is smaller than $\Lambda$. Interestingly, in this present study, we find that by defining $\alpha=\left(\mathrm{PF}_{\text {opt }} / \kappa_{l}\right) T$, we can have a direct relation of $Z T_{\mathrm{opt}}$ with $P F_{\text {opt }}$ through Eq. 15 . Note that $W_{0}(\alpha)$ monotonically increases with $\alpha$, as shown in Fig. 4 in Appendix B. It is important to point out that the factor $(L / \Lambda)^{d-3}$ is not only the enhancement factor of the $\mathrm{PF}_{\text {opt }}$, but also of $Z T_{\mathrm{opt}}$ for the low-dimensional semiconductors.

\section{The universal curve}

Let us now compare the $Z T_{\text {opt }}$ formula with various experimental data. In Fig. 3, we plot theoretical $Z T_{\text {opt }}$ (solid curve) as a function of $\alpha$ [Eq. (15)]. Here $Z T_{\mathrm{opt}}$ merely depends on $\mathrm{PF}_{\text {opt }}, \kappa_{l}$, and $T$, despite of the fact that the PF and $Z T$ are optimized at different chemical potentials, i.e., $\eta_{\mathrm{opt}}^{\mathrm{PF}}=0$ and $\eta_{\mathrm{opt}}^{Z T}=-W_{0}(\alpha)$, re- 
spectively. Hence, $Z T_{\mathrm{opt}}$ from various materials with different dimensions can be compared directly with the theoretical curve. The experimental data (symbols) in Fig. 3 are extracted from plots of $Z T_{\mathrm{opt}}, \mathrm{PF}_{\mathrm{opt}}$, and $\kappa_{l}$ in Refs. 16 20] by using digitizer software. These data include 1D Bi nanowires of different diameters $(\sim 38$ $290 \mathrm{~nm}$ ) along with bulk 3D Bi at room temperature [16, $2 \mathrm{D} \mathrm{PbTe}$ quantum wells of different thicknesses $(\sim 1.9$ $4.0 \mathrm{~nm}$ ) along with 3D PbTe at room temperature [17, also $3 \mathrm{D} \mathrm{Pb}_{0.98} \mathrm{Na}_{0.02} \mathrm{Te}$ [18, $3 \mathrm{D} \mathrm{FeNb}{ }_{0.8} \mathrm{Ti}_{0.2} \mathrm{Sb}$ [19], and 3D $\mathrm{Mg}_{2} \mathrm{Sn}_{0.78} \mathrm{Ge}_{0.2} \mathrm{Sb}_{0.02}$ [20] at different temperatures ( 300-1100 K).

As can be seen in Fig. 3 all experimental data tend to fit the theoretical curve from Eq. (15). The values of $Z T_{\text {opt }}$ monotonically increase as a function of $\alpha$ and thus we can say that any semiconductor should have the material parameter $\alpha>4.5$ to obtain $Z T_{\text {opt }}>2$. At smaller $\alpha$ values (higher $T$ or higher $\mathrm{PF}_{\mathrm{opt}}$ ), we have $\eta_{\mathrm{opt}}^{Z T} \sim \eta_{\mathrm{opt}}^{\mathrm{PF}}$, especially around $\alpha<0.3$. In this case, $Z T_{\mathrm{opt}} \sim\left(\mathrm{PF}_{\mathrm{opt}} / \kappa_{l}\right) T$ [see the dotted line in Fig. 3. On the other hand, at larger $\alpha$, we have $\eta_{\mathrm{opt}}^{Z T}<\eta_{\mathrm{opt}}^{\mathrm{PF}}$ that eventually results in a nonlinear function of $Z T_{\text {opt }}$ versus $\left(\mathrm{PF}_{\mathrm{opt}} / \kappa_{l}\right) T$. The main benefit of using the universal curve in Fig. 3 is that it provides a new way to directly calculate $Z T_{\text {opt }}$ from $\mathrm{PF}_{\mathrm{opt}}$ and $\kappa_{l}$ without any necessity to check the electron thermal conductivity $\kappa_{e}$ nor the optimum chemical potential $\eta_{\mathrm{opt}}^{Z T}$.

\section{CONCLUSION}

We have shown that the simple analytical formulas [Eq. [15] ] based on the one-band model can directly relate the optimum figures of merit $Z T_{\mathrm{opt}}$ and the optimum power factors $\mathrm{PF}_{\mathrm{opt}}$ of semiconductors with different dimensions. By introducing the material parameter $\alpha=\left(\mathrm{PF}_{\mathrm{opt}} / \kappa_{l}\right) T$, we can obtain the universal curve of $Z T_{\text {opt }}$ combining both bulk and low-dimensional semiconductors, in which $Z T_{\mathrm{opt}}$ monotonically increases as a function of $\alpha$. Since this approach reduces parameters such as $\kappa_{e}$ and $\eta_{\mathrm{opt}}^{Z T}$ in the calculation of $Z T_{\mathrm{opt}}$, we believe that it will help researchers better identify new thermoelectric materials in the future.

\section{ACKNOWLEDGMENTS}

This work is dedicated to the late Prof. M. S. Dresselhaus. N.T.H. and A.R.T.N thank Dr. E. H. Hasdeo for fruitful discussions and acknowledge the financial support from the Interdepartmental Doctoral Degree Program for Multi-dimensional Materials Science Leaders, Tohoku University. R.S. acknowledges JSPS KAKENHI Grant Numbers JP25107005 and JP25286005.

\section{Appendix A: The scattering coefficient $C$}

\section{Defining $C$ from Fermi's golden rule}

Fermi's golden rule gives the scattering rate of transitions between discrete states $|\mathbf{k}\rangle$ and $\left|\mathbf{k}^{\prime}\right\rangle$ as follows [21]

$$
\frac{1}{\tau\left(\mathbf{k} \rightarrow \mathbf{k}^{\prime}\right)} \approx \frac{2 \pi}{\hbar}\left|\left\langle\mathbf{k}^{\prime}|V| \mathbf{k}\right\rangle\right|^{2} \delta\left[E(\mathbf{k})-E\left(\mathbf{k}^{\prime}\right)\right],
$$

where $\hbar$ is the Planck constant, $V$ is the perturbation potential, $\delta$ is the Dirac-delta function, and $E$ is the energy dispersion. The general scattering rate is given by the product $2 \pi / \hbar$ times the square of transition matrix element square, times a Dirac-delta function. For the one-band model, the scattering rate will be between states within parabolic energy band, where a continuum of states exist. In this case, the final scattering rate will be obtained by summation over all relevant states,

$$
\begin{aligned}
\frac{1}{\tau(\mathbf{k})} & =\sum_{\mathbf{k}^{\prime}} \frac{1}{\tau\left(\mathbf{k} \rightarrow \mathbf{k}^{\prime}\right)} \\
& =\frac{2 \pi}{\hbar} \sum_{\mathbf{k}^{\prime}}\left|\left\langle\mathbf{k}^{\prime}|V| \mathbf{k}\right\rangle\right|^{2} \delta\left[E(\mathbf{k})-E\left(\mathbf{k}^{\prime}\right)\right]
\end{aligned}
$$

As an example, consider the scattering rate between electron states in the conduction band due to a point scatterer in a 3D semiconductor. Let us consider a perturbing potential as $V(\mathbf{r})=V_{0} \delta(\mathbf{r})$ for short-range interactions, where $V_{0}$ is constant in units of $\mathrm{Jm}^{3}$. The matrix element between electronic states $|\mathbf{k}\rangle$ and $\left|\mathbf{k}^{\prime}\right\rangle$ can be obtained as 22.

$$
\begin{aligned}
& \left|\left\langle\mathbf{k}^{\prime}\left|V_{0} \delta(\mathbf{r})\right| \mathbf{k}\right\rangle\right| \\
& =\int \mathrm{d}^{3} \mathbf{r}\left(\frac{e^{-i \mathbf{k}^{\prime} \cdot \mathbf{r}}}{\sqrt{\Omega}}\right) V_{0} \delta(\mathbf{r})\left(\frac{e^{+i \mathbf{k}^{\prime} \cdot \mathbf{r}}}{\sqrt{\Omega}}\right)=\frac{V_{0}}{\Omega},
\end{aligned}
$$

where $\Omega$ is the volume of the system. After substituting the matrix element in Eq. A3 into Eq. A2, the scattering rate can be written as

$$
\frac{1}{\tau(\mathbf{k})}=\frac{2 \pi}{\hbar}\left(\frac{V_{0}}{\Omega}\right)^{2} \sum_{\mathbf{k}^{\prime}} \delta\left[E(\mathbf{k})-E\left(\mathbf{k}^{\prime}\right)\right] .
$$

By using the carrier density of states (DOS), defined as $\mathcal{D}(E)=\frac{2}{\Omega} \sum_{\mathbf{k}} \delta[E-E(\mathbf{k})]$ in units of $\mathrm{J}^{-1} \mathrm{~m}^{-3}$, where the factor 2 accounts for the spin degeneracy, Eq. A4 is now expressed as

$$
\frac{1}{\tau(E)}=\frac{\pi V_{0}^{2}}{\hbar \Omega} \mathcal{D}(E)
$$

This example shows an important result indicating that the scattering rate for the continuum of states is in general proportional to the DOS, while the strength of scattering increases with the square of the scattering 
potential. The carrier relaxation time $\tau(E)$ is thus inversely proportional to the carrier DOS:

$$
\tau(E)=C \mathcal{D}^{-1}(E)
$$

where $C=\hbar \Omega /\left(\pi V_{0}^{2}\right)$ is the scattering coefficient in units of $\mathrm{W}^{-1} \mathrm{~m}^{-3}$. Note that according to Fermi's golden rule, the coefficient $C$ can be a constant value when the matrix element is approximately constant.

\section{Calculating $C$ from experimental data}

Here we derive a formula of the coefficient $C$ considering a parabolic band for any semiconductor so that $C$ can be calculated from experimental data. The carrier relaxation time $\tau(E)$ and the density of states $\mathcal{D}(E)$ per unit volume are, respectively, defined by [14, 22 ]

$$
\begin{aligned}
\tau(E) & =\tau_{0}\left(\frac{E}{k_{B} T}\right)^{r}, \\
\mathcal{D}(E) & =\frac{\left(2 m / \hbar^{2}\right)^{d / 2} E^{d / 2-1}}{L^{3-d} 2^{d-1} \pi^{d / 2} \Gamma\left(\frac{d}{2}\right)},
\end{aligned}
$$

where $k_{B}$ is the Boltzmann constant, $T$ is the average absolute temperature, $\tau_{0}$ is the carrier relaxation time coefficient, $r$ is a characteristic exponent, $d=1,2,3$ denotes the dimension of the system, $m$ is the carrier effective mass, and $L$ is the confinement length for a particular material dimension. For a given $\tau(E)$, the carrier mobility is defined by

$$
\mu=\frac{q\langle\langle\tau(E)\rangle\rangle}{m} .
$$

The average relaxation time is defined by 22$]$

$$
\langle\langle\tau(E)\rangle\rangle \equiv \frac{\langle E \tau(E)\rangle}{\langle E\rangle}=\tau_{0} \frac{\Gamma\left(\frac{5}{2}+r\right)}{\Gamma\left(\frac{5}{2}\right)},
$$

where $\Gamma$ is the Gamma function. From Eqs. A7), A9, and $\mathrm{A} 10$, the carrier relaxation time $\tau(E)$ can be rewritten as

$$
\tau(E)=\frac{\mu m \Gamma\left(\frac{5}{2}\right)}{q \Gamma\left(\frac{5}{2}+r\right)}\left(\frac{E}{k_{B} T}\right)^{r} .
$$

We assume that the acoustic phonon scattering is the main carrier scattering mechanism at the room temperature, i.e., $\tau(E) \propto \mathcal{D}(E)^{-1}$ 12, 22. From Eqs. A8, A11 and $\tau(E) \propto \mathcal{D}(E)^{-1}$, we obtain $r=$ $1-d / 2$ for the system with the dimension $d$. By using $r=1-d / 2$, from Eqs. A6, (A8), and A11), the coefficient $C$ can be written as

$$
\begin{aligned}
C & =\tau(E) \mathcal{D}(E) \\
& =\frac{2 \mu m \Gamma\left(\frac{5}{2}\right)}{q k_{B} T L^{3-d} \Gamma\left(\frac{7-d}{2}\right) \Gamma\left(\frac{d}{2}\right)}\left(\frac{m k_{B} T}{2 \pi \hbar^{2}}\right)^{d / 2} .
\end{aligned}
$$

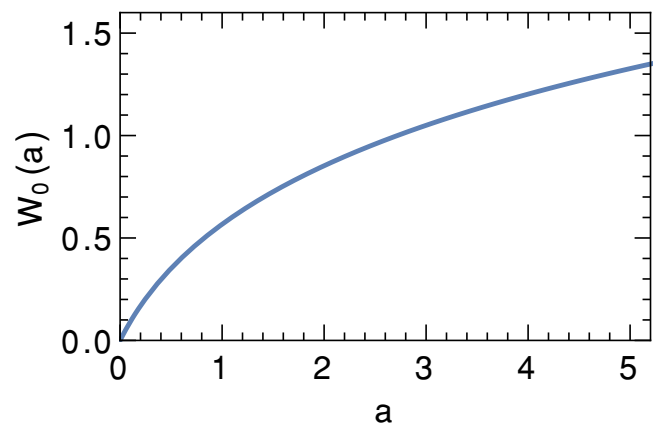

FIG. 4. The real principal branch of the $W$ function in the case of $a \in[0, \infty)$.

After substituting the thermal de Broglie wavelength $\Lambda=\left(2 \pi \hbar^{2} / m k_{B} T\right)^{1 / 2}$ into Eq. A12, the coefficient $C$ is given by

$$
C=\frac{2 \mu m}{q k_{B} T L^{3}}\left(\frac{L}{\Lambda}\right)^{d} \frac{\Gamma\left(\frac{5}{2}\right)}{\Gamma\left(\frac{7-d}{2}\right) \Gamma\left(\frac{d}{2}\right)} .
$$

Equation A13 is useful to calculate the coefficient $C$ from $\mu$ and $m$, which can be obtained from experimental data. For example, in the 3D $(d=3)$ n-type $\mathrm{Bi}_{2} \mathrm{Te}_{2.7} \mathrm{Se}_{0.3}$ [13, at room temperature $(T=298 \mathrm{~K})$ and doping concentration on the order of $10^{19} \mathrm{~cm}^{3}$, the carrier mobility and the carrier effective mass are $\mu=173$ $\mathrm{cm}^{2} / \mathrm{Vs}$ and $m=1.12 m_{0}$, respectively, where $m_{0}$ is the free electron mass. From Eq. A13, we obtain the $C$ value of about $1.18 \times 10^{33} \mathrm{~W}^{-1} \mathrm{~m}^{-3}$ and correspondingly the average relaxation time is about $0.1 \mathrm{ps}$.

\section{Appendix B: The Lambert $W$ function}

The Lambert $W$ function is defined as a multivalued complex function that satisfy the following equation:

$$
W(\alpha)=\alpha e^{-W(\alpha)}, \quad \alpha \in \mathbb{C} .
$$

Equation (B1) always has an infinite number of solution in the complex Liemann plane, hence the multivaluedness of the $W$ function. These solutions are indexed by the integer variable $j$ and are called the branches of the $W$ function, $W_{j}$, for $j \in \mathbb{Z}$. In particular, the solutions of Eq. (B1) in the calculation of $Z T_{\text {opt }}$ correspond to $\alpha \in[0, \infty)$. In this case there can be a real solution, corresponding to the principal branch of the $W$ function, i.e. $W_{0}(\alpha) \in[0, \infty)$.

The $W_{0}$ function can be written in terms of series expansion as follows 23,

$$
\begin{aligned}
W_{0}(\alpha)= & \sum_{n=1}^{\infty} \frac{(-n)^{n-1}}{n !} \alpha^{n} \\
= & \alpha-\alpha^{2}+\frac{3}{2} \alpha^{3}-\frac{8}{3} \alpha^{4}+\frac{125}{24} \alpha^{5} \\
& -\frac{54}{5} \alpha^{6}+\frac{16807}{720} \alpha^{7}+\cdots
\end{aligned}
$$


Figure 4 shows $W_{0}(\alpha)$ as a function of $\alpha$ when $\alpha \in[0, \infty)$.

[1] L. E. Bell, Cooling, heating, generating power, and recovering waste heat with thermoelectric systems, Science 321, 1457 (2008).

[2] J. P. Heremans, M. S. Dresselhaus, L. E. Bell, and D. T. Morelli, When thermoelectrics reached the nanoscale, Nature Nanotechnol. 8, 471 (2013).

[3] C. B. Vining, An inconvenient truth about thermoelectrics, Nature Mater. 8, 83 (2009).

[4] L. D. Hicks and M. S. Dresselhaus, Effect of quantumwell structures on the thermoelectric figure of merit, Phys. Rev. B 47, 12727 (1993).

[5] L. D. Hicks and M. S. Dresselhaus, Thermoelectric figure of merit of a one-dimensional conductor, Phys. Rev. B 47, 16631 (1993).

[6] L. D. Hicks, T. C. Harman, X. Sun, and M. S. Dresselhaus, Experimental study of the effect of quantum-well structures on the thermoelectric figure of merit, Phys. Rev. B 53, R10493 (1996).

[7] N. T. Hung, E. H. Hasdeo, A. R. T. Nugraha, M. S. Dresselhaus, and R. Saito, Quantum effects in the thermoelectric power factor of low-dimensional semiconductors, Phys. Rev. Lett. 117, 036602 (2016).

[8] G. D. Mahan and J. O. Sofo, The best thermoelectric, Proc. Natl. Acad. Sci. U.S.A. 93, 7436 (1996).

[9] C. Jeong, R. Kim, and M. S. Lundstrom, On the best bandstructure for thermoelectric performance: A landauer perspective, J. Appl. Phys. 111, 113707 (2012).

[10] R. P. Chasmar and R. Stratton, The thermoelectric figure of merit and its relation to thermoelectric generators, J. Electron. Control 7, 52 (1959).

[11] H. J. Goldsmid, Introduction to Thermoelectricity (Springer-Verlag, Berlin Heidelberg, 2010).

[12] J. Zhou, R. Yang, G. Chen, and M. S. Dresselhaus, Optimal bandwidth for high efficiency thermoelectrics, Phys. Rev. Lett. 107, 226601 (2011).

[13] W. S. Liu, Q. Zhang, Y. Lan, S. Chen, X. Yan, Q. Zhang, H. Wang, D. Wang, G. Chen, and Z. Ren,
Thermoelectric property studies on $\mathrm{Cu}$-doped n-type $\mathrm{Cu}_{x} \mathrm{Bi}_{2} \mathrm{Te}_{2.7} \mathrm{Se}_{0.3}$ nanocomposites, Adv. Energy Mater. 1, 577 (2011).

[14] N. T. Hung, A. R. T. Nugraha, E. H. Hasdeo, M. S. Dresselhaus, and R. Saito, Diameter dependence of thermoelectric power of semiconducting carbon nanotubes, Phys. Rev. B 92, 165426 (2015).

[15] C. Kittel and H. Kroemer, Thermal physics (W. H. Freeman, 1980).

[16] J. Kim, S. Lee, Y. M. Brovman, P. Kim, and W. Lee, Diameter-dependent thermoelectric figure of merit in single-crystalline Bi nanowires, Nanoscale 7, 5053 (2015).

[17] T. C. Harman, D. L. Spears, and M. J. Manfra, High thermoelectric figures of merit in $\mathrm{PbTe}$ quantum wells, J. Electron. Mater. 25, 1121 (1996).

[18] L. D. Zhao, H. J. Wu, S. Q. Hao, C. I. Wu, X. Y. Zhou, K. Biswas, J. Q. He, T. P. Hogan, C. Uher, C. Wolverton, V. P. Dravid, and M. G. Kanatzidis, All-scale hierarchical thermoelectrics: MgTe in PbTe facilitates valence band convergence and suppresses bipolar thermal transport for high performance, Energy Environ. Sci. 6, 3346 (2013).

[19] C. Fu, T. Zhu, Y. Liu, H. Xie, and X. Zhao, Band engineering of high performance p-type FeNbSb based half-Heusler thermoelectric materials for figure of merit ZT > 1, Energy Environ. Sci. 8, 216 (2015).

[20] W. Liu, J. Zhou, Q. Jie, Y. Li, H. S. Kim, J. Bao, G. Chen, and Z. Ren, New insight into the material parameter B to understand the enhanced thermoelectric performance of $\mathrm{Mg}_{2} \mathrm{Sn}_{1-x-y} \mathrm{Ge}_{x} \mathrm{Sb}_{y}$, Energy Environ. Sci. 9, 530 (2016).

[21] J. M. Ziman, Principles of the Theory of Solids (Cambridge University Press, New York, 1972).

[22] M. Lundstrom, Fundamentals of carrier transport (Cambridge University Press, New York, 2009).

[23] C. Carathéodory, Theory of Functions of a Complex Variable (AMS Chelsea Publishing, New York, 1954). 